\title{
Panorama succinct des sciences psychologiques entre 1575 et 1625
}

Par Jean Starobinski

Le mot psychologie fait son apparition au XVI ${ }^{\mathrm{e}}$ siècle. Il est signalé dans le titre d'un traité latin, non retrouvé, de Marko Marulic (1450-1524): Psichiologia de ratione animae. Un examen attentif des œuvres de Melanchthon ne permet pas de confirmer sa participation, souvent alléguée, à l'invention et à la diffusion du terme. Celui-ci est utilisé par Johannes Thomas Freige (Freigius) dans le Catalogus locorum communium qui figure au début de son Ciceronianus (Bâle, 1575). Il apparaît dans le titre du livre de Rudolf Goeckel (1547-1628) (Goclenius): Psychologia, hoc est de hominis perfectione, animo et imprimis ortu eius, commentationes ac disputationes (Marburg, 1590); le même auteur fait paraître, en 1596, un De praecipuis materiis psychologicis; puis, en 1597, un ouvrage intitulé Authores varii de psychologia. Otto Casmann (1562-1607), disciple de Goclenius, reprend le terme dans un livre portant le titre: Psychologia anthropologica, sive animae humanae doctrina (Hanovre, 1594). Le terme correspond-il à la création d'un nouveau concept? Si tel eût été le cas, Goclenius l'eût fait figurer dans son Lexicon Philosophicum (1613): or il en est absent ${ }^{1}$. Ce n'est pas le nom d'une nouvelle discipline, ni l'indice d'un découpage original parmi les objets d'intérêt. Nous avons affaire ici à un néologisme savant, peut-être induit par l'emploi fréquent du mot Physiologia dans les traités médicaux. De même que Physiologia équivaut au latin De natura, Psychologia équivaut à De anima ${ }^{2}$. Ainsi la Psychologia de Goclenius expose le débat sur l'immortalité de l'âme et confronte les thèses opposées sur l'introduction de l'âme dans le corps. Le livre est un receuil de dissertations empruntées à une quinzaine d'auteurs. «Entre ceux qui soutiennent qu'elle est créée directement par Dieu, et ceux qui prétendent qu'elle est transmise des parents aux enfants, Goclenius qui écrit à peine quelques lignes pour son compte, garde un doute prudent: 'Il vaut mieux, dit-il, chercher à savoir comment l'âme sortira du corps sans souillure, que comment elle y a pénétré'. ${ }^{3}$ Quant à Casmann, c'est un théologien attiré par les grandes systématisations, et qui complète sa psychologie par un ouvrage consacré à la description du corps (Anthropologiae pars secunda, i.e. de fabrica humani corporis methodice descripta, Hanovre, 1596) et par une Angelographia (Francfort, 1597), puis par une Somatologia physica generalis. 
Les historiens modernes de la psychologie signalent habituellement l'emploi du terme dans les titres de Goclenius et de Casmann, philosophes de Marburg, mais ils se hâtent de les quitter: "The school was so far limited to theological interests that its doctrines need not be discussed», écrit George Sidney Brett ${ }^{4}$.

De fait, si nous interrogeons la période finale de la Renaissance en y reportant le concept moderne de psychologie, nous ne trouverons aucune «science » distincte qui lui corresponde. Pourtant la matière psychologique est ample. Il nous faudra prélever les pages qui nous intéressent dans les sommes du savoir, chez les philosophes, chez les théologiens, chez les médecins. La psychologie n'occupe pas un champ spécifique et ne constitue pas une discipline isolable. Elle fait partie intégrante de plusieurs édifices systématiques, où les mêmes propositions se répètent, mais à d'autres places. Bref, nous ne pouvons que construire une «psychologie de la Renaissance», par abstraction, en choisissant a posteriori les chapitres qui correspondent aux intérêts de notre psychologie et de notre psychiatrie.

Scipion Du Pleix (1569-1661) explique fort bien, dans son Corps complet de philosophie (1607) que l'étude de l'âme appartient à la physique, dans la mesure où l'âme est considérée dans ses effets corporels, mais que cette étude relève de la métaphysique, dans la mesure où l'essence de l'âme est envisagée indépendamment des liens avec le corps. La théologie, et plus particulièrement la pneumatologie, auront pour tâche d'établir les vérités touchant l'origine et les fins de l'âme, ainsi que son rang dans les hiérarchies spirituelles (l'angélographie et la démonologie devant compléter le tableau des créatures). L'étude des passions, vices, vertus est du ressort de l'éthique et de la théologie morale: la casuistique, attentive à la distinction entre péché volontaire et faute involontaire, contribuera à développer un certain raffinement "psychologique». Enfin, au carrefour de la métaphysique, de la physique et de la logique, se pose la question des pouvoirs intellectuels de l'homme, des voies d'accès de la connaissance, de ses limites, etc.: la "psychologie» se développe en ce qui pour nous est une «épistémologie».

La Physiologia 5 (1542), première partie de l'Universa Medicina de Jean Fernel (1485-1557), constamment rééditée jusqu'en 1680, montre très clairement comment le savoir fait appel à la notion d'âme dans le domaine de la "physique». La systématisation aristotélo-galénique dont Fernel, malgré certains critiques de détail, apporte une excellente illustration se perpétuera, pratiquement inchangée, jusqu'à la critique cartésienne des «facultés occultes». Dans l'intervalle, toutes les Physiologies se ressemblent. A près de cent ans de distance, Daniel Sennert ${ }^{6}$ (1572-1637) s'exprime sur les facultés de l'âme comme Fernel. Et sur ce point les vulgarisateurs - tels Pierre de La Primaudaye dans son Académie françoise (1577-1596) - en disent autant que les médecins. 
La psychologie s'expose ${ }^{7}$ :

1) Dans la nomenclature hiérarchisée des fonctions et des facultés de l'âme, selon la tripartition traditionnelle.

Ame
végétative $\quad\left\{\begin{array}{l}\text { faculté nutritive } \\ \text { faculté d'accroissement } \\ \text { faculté générative }\end{array} \quad \begin{array}{l}\begin{array}{l}\text { faculté attrayante } \\ \text { faculté rétentive } \\ \text { faculté concoctive } \\ \text { faculté expultrice }\end{array} \\ \end{array}\right.$

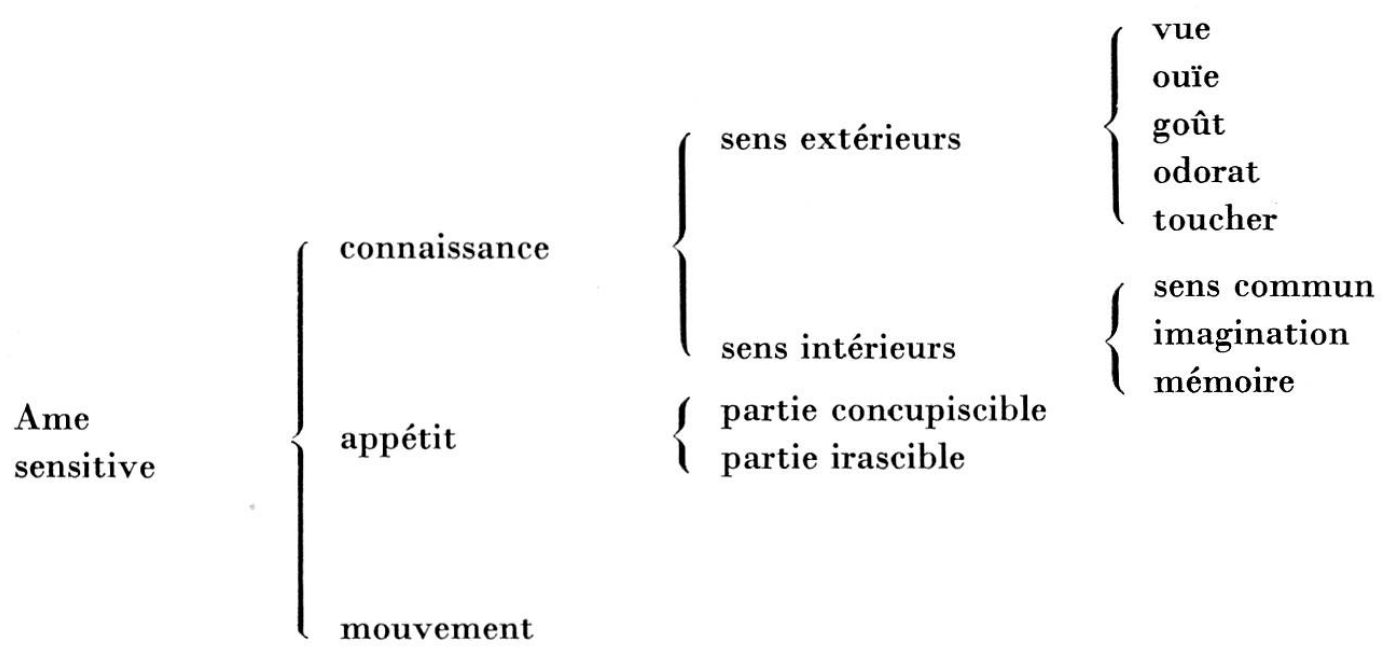

Ame
intellectuelle $\left\{\begin{array}{l}\text { entendement } \\ \text { volonté } \\ \text { mémoire }\end{array} \quad\left\{\begin{array}{l}\text { patient } \\ \text { agent } \\ \text { faculté composante } \\ \text { faculté ratiocinante } \\ \text { (inférer, distinguer, } \\ \text { choisir) }\end{array}\right.\right.$

Les auteurs ne manquent pas d'observer comme Du Pleix que «ce qui est âme sensitive aux bêtes n'est que faculté de l'âme intellectuelle à l'homme». 
Mais la psychologie s'expose à nouveau:

2) Dans l'énoncé des conditions matérielles nécessaires à l'exercice des fonctions de l'âme.

Il faut qu'à partir des aliments et de l'air respiré se forme l'esprit naturel (au foie), l'esprit vital (au cœur), l'esprit animal (au cerveau). Il faut que les quatre humeurs (sang, phlegme, bile jaune, bile noire) soient heureusement tempérées. Les diverses dyscrasies modifieront la chaleur des organes ou la qualité, la quantité, la densité des esprits qui, entre l'âme et le corps, jouent un rôle instrumental. En stricte orthodoxie, l'âme immatérielle et immortelle ne peut être menacée par l'intempérie humorale que dans ses manifestations corporelles, et non dans son existence même. Mais il va sans dire que le discours physiologique, fondé sur les causes naturelles, tend à se donner le plus large pouvoir explicatif, et à éviter, sous les apparences du respect à l'égard des vérités révélées, le recours au «principe immatériel». La tradition aristotélo-galénique, dont la science médicale est dépositaire, peut s'énoncer sans recourir à Dieu ni au concept d'âme immatérielle: la vie psychologique de l'individu - sentiment, pensée, volonté sera un effet des forces de la Nature. Dès lors que la matière plus ou moins «subtilisée» est cause nécessaire du mouvement, de la perception, etc., pourquoi n'en' serait-elle pas aussi la cause suffisante? Même si tous les médecins ne le pensent pas, ils seront toujours suspects d'avoir cette arrière-pensée. D'où, dans la littérature de l'époque, tant de personnages de médecins qui jouent des rôles d'athées plus ou moins camouflés ${ }^{8}$.

Si la tradition galénique autorise à construire la psychologie (la théorie des sensations, de l'imagination, des passions) sur une base somatique et humorale, si elle attribue aux tempéraments du corps une valeur déterminante quant aux «mœurs de l'âme», rappelons qu'elle ne méconnaît nullement l'influence exercée par les passions sur le corps. Tous les traités médicaux comportent une section consacrée à l'hygiène, et l'on y trouve infailliblement la mention de l'effet exercé sur la santé du corps par les «six choses non-naturelles». Celles-ci sont: l'air, la nourriture et les boissons, la veille et le sommeil, le mouvement et le repos, la réplétion et l'évacuation, les passions de l'âme. Qu'un dérèglement intervienne dans l'une ou l'autre de ces six catégories, le corps et les humeurs en pâtiront. Toute recherche étiologique doit y remonter: et, comme les passions de l'âme figurent dans la liste, la possibilité d'une étiologie «psychosomatique» est pleinement envisagée. Dans nombre de traités, les passions de l'âme sont spécifiées; elles sont au nombre de six: metus, mestitia, ira, gaudium (dont l'influence peut être favorable), agonia, verecundia ${ }^{9}$. Ce clavier passionnel offrira aussi des ressources thérapeutiques: dans la mesure où la crainte (metus) refoidit, elle sera 
mise en œuvre avec profit dans les «intempéries chaudes»; dans la mesure où la colère échauffe, le médecin pourra chercher à la provoquer chez des patients en état de torpeur ${ }^{10}$. Les passions entrent en compte dans le traitement des contraires par les contraires.

Tel est le cadre général dans lequel s'inscrit la théorie psychologique de l'époque: cadre suffisamment solide pour rester partout incontesté, suffisamment accueillant pour admettre les variantes, les retouches locales, les apports empiriques ${ }^{11}$, les investigations monographiques. Monotone et limitée dans ses exposés doctrinaux, cette psychologie comporte de nombreux degrés de liberté dans ses applications pratiques. Ainsi Felix Platter (1536-1614) distribue les tomes de sa Praxis Medica (Bâle, 1602-1608) selon un principe symptomatologique: 1) Lésion des fonctions, 2) Douleurs, 3) Vices du corps et des excrétions. Le tome consacré aux lésions des fonctions contient 150 pages consacrées aux maladies mentales: c'est le tableau le plus important qui en ait été dressé avant l'essor de la spécialité psychiatrique au $\mathrm{XIX}^{\mathrm{e}}$ siècle. Il atteste une attention aiguë aux désordres du comportement. On a pu dire qu'il portait la marque de l'esprit «baroque ${ }^{8}$, car il fait une très large place à la description de phénomènes excessifs: torpeurs prolongées, ivresses pathologiques, fureurs délirantes: la description plattérienne de la nymphomanie, "fureur utérine», sera maintes fois citée par les auteurs des siècles suivants. Le système préétabli accueille ici les observations individuelles, et s'ouvre à la classification descriptive des symptômes.

Moins discursive, la littérature des Consilia, des Observationes, des Letterae Medicinales connaît un large essor ${ }^{12}$. Certains des cas décrits relatent des troubles psychiques extraordinaires: ce sont parfois de véritables nouvelles médicales, plus développées que les observations rapportées par les Epidémies hippocratiques. Une cause naturelle en donne généralement la clé satisfaisante. Et ces histoires seront reversées dans les traités systématiques, à titre d'exemples illustratifs: on les citera scrupuleusement jusqu'au début du $\mathrm{XIX}^{\mathrm{e}}$ siècle, sur la foi de la tradition.

D'autres auteurs, moins dociles à l'égard de l'enseignement canonique, contestent certaines hypothèses étiologiques généralement admises. Mais il ne font qu'opérer un déplacement d'accent à l'intérieur du système des causes prévues par la doctrine aristotélo-galénique. Ainsi Charles Lepois (Piso) (1563-1636) attribue à un flux séreux (colluvies serosa) survenant au niveau des racines cérébrales, les phénomènes d'hystérie et d'épilepsie que l'on assignait à des sympathies organiques, à des vapeurs montées du bas-ventre, à la suffocation de l'utérus. Lepois, bien souvent, ne fait que retrouver Galien et Hippocrate ${ }^{13}$, en écartant les hypothèses surajoutées par Fernel. 
A peu près intangible dans ses présupposés téléologiques, dans sa physique des éléments et des qualités, le système aristotélo-galénique propose des questions partout où, dans son développement explicatif, il passe d'une catégorie d'objets à une autre, partout où il établit des indices et des corrélations d'un domaine à l'autre. L'attention se fixe sur les problèmes qui surviennent aux points de transition: de l'ordre des humeurs à celui des émotions, de l'ordre des sensations à celui des pensées; de l'ordre de l'imagination à celui des stigmates corporels ou des maladies. Ces questions seront matière à disputes, à thèses ${ }^{14}$, à monographies plus ou moins développées. L'intention de ces innombrables écrits n'est pas de modifier le savoir, de le faire progresser en recourant à de nouveaux principes explicatifs, mais d'accumuler les preuves convaincantes, d'augmenter et de consolider la démonstration des enchaînements ou la table des indices. Citons quelques-uns des sujets les plus fréquemment traités: la puissance de l'imagination, l'effet de la musique sur l'âme et sur le corps (notamment dans le tarantisme), l'étendue des pouvoirs de la fascination, la physiognomonie des tempéraments et des passions, l'interprétation des rêves comme révélateurs des maladies du corps, et surtout: le rôle des humeurs, de leur crase, de leurs altérations dans la détermination des traits caractériels, des qualités et des défauts de l'esprit. En ce domaine, l'héritage de Ficin est particulièrement sensible: parmi les humeurs, la mélancolie est l'objet d'une attention privilégiée. Les états extrêmes du comportement, stupeur craintive ou délire maniaque, sont attribués aux variations caloriques ou qualitatives de la bile noire. Mais une complexion modérément mélancolique, tout en favorisant la tristesse, va de pair avec les plus hautes qualités intellectuelles: la prudence politique, l'inspiration poétique, la pénétration contemplative, la sagacité scientifique. Si ambigus que soient les dons de Saturne ${ }^{15}$, il est bienséant d'avoir cette planète dans son horoscope, de porter le noir, de hanter les lieux écartés, de chercher la guérison sous d'autre cieux, et d'en revenir désabusé. Le type du malcontent traveller mélancolique a été décrit comme l'un des représentants de la «maladie élisabéthaine ». ${ }^{16}$ La complexion mélancolique est alléguée pour expliquer la folie du Tasse, la retraite de Charles-Quint, les travers de Philippe II. Ronsard se déclare mélancolique. Montaigne dit avoir entrepris les Essais "par une humeur mélancolique». Jaques (dans As you like it), Hamlet, Don Quichotte sont des mélancoliques... Les traités médicaux les plus fameux sont ceux de Timothy Bright (vers 1551-1615), (Londres, 1586), d'André Du Laurens (1558-1609) (Paris, 1597), de Jacques Ferrand (De la maladie d'amour, ou mélancholie érotique, Paris, 1623). Ces ouvrages sont l'occasion d'amples citations empruntées aux anciens; les cas relatés, les guérisons mentionnées sont pour la plupart d'origine livresque. Dans l'Anatomy of Melancholy (1621) de 
Robert Burton (1577-1640), la monographie érudite prend les dimensions d'un vaste édifice de savoir et de sagesse. Le succès du livre est grand: le texte se modifie et se développe au gré de six éditions assurées par l'auteur. Robert Burton n'est pas médecin : c'est un théologien dont l'existence entière est liée à Brasenose College. Son livre toutefois doit son architecture aux subdivisions proposées par la science médicale. D'une première partie qui traite des causes, des symptômes et du pronostic de la mélancolie, l'on passe à une seconde partie consacrée aux remèdes, puis à une partie finale occupée par l'examen de deux variétés remarquables de la maladie: la mélancolie amoureuse et la mélancolie religieuse. Cette structure fondamentale - garantie par la solide organisation du savoir médical traditionnel - constitue l'ossature logique du livre: elle permet les accrétions, les digressions, les enchaînements latéraux. Il en résulte une bizarre somme sur la condition humaine: l'auteur, sous le masque de Democritus Junior, se présente lui-même comme un mélancolique ${ }^{17}$. S'il écrit un livre sur un tel sujet, c'est pour «se gratter où il lui cuit». Il se donne du moins, pour en rire, le spectacle de la folie du genre humain, - folie due, elle aussi, à l'omniprésence de la mélancolie. L'épître au lecteur est l'occasion de développer une utopie politicosociale, en opposition à la folie du monde. Les considérations sur les effets de l'air est l'occasion d'une digression géographique et cosmologique; l'exposé sur la mélancolie religieuse se prolonge en une attaque contre les sectaires inspirés ou visionnaires, contre les enthousiastes et les faux prophètes. En dépit de son immense appareil ornemental de citations, le livre de Burton n'est pas un simple inventaire savoureusement énoncé: il porte la marque d'une personnalité, il prend parti dans des questions d'actualité. La nouveauté de l'ouvrage ne réside pas dans la théorie psychologique elle-même, mais dans le fait qu'un si ample tableau du monde humain s'organise et se développe à partir de la psychophysiologie des tempéraments et des humeurs: la vision du monde et de l'homme tend à se «psychologiser». La «complexion» de l'homme est le terme de référence permanent, même si les entités spirituelles - Dieu, le diable, l'âme humaine - ne sont pas oubliées. La «complexion» est principe d'individuation, au moment où l'individualisme prend son essor.

Certes, d'autres théologiens pourront développer des vues «psychologiques» dans une perspective moins médicale, et sans remonter aux tempéraments du corps. Quand la morale fait l'inventaire des vertus et des vices, elle décrit des conduites spécifiques, et les auteurs se trouvent amenés à tracer des «caractères» partiellement inspirés par les modèles littéraires de l'antiquité. Ces descriptions, qui ont un aspect synthétique, proposent des types stables, mais les nuancent en les multipliant. Ainsi fait Joseph Hall (1574-1656), évêque d'Exeter, dans ses 
Characters of Virtues and Vices (1608), ou Nicolas Coëffeteau dans son Tableau des passions humaines (1620): livres à succès, où romanciers et dramaturges vinrent souvent chercher leurs personnages.

Entre théologiens et médecins, la bile noire marque une frontière litigieuse dans le débat qui les oppose au sujet de la sorcellerie et de la possession démoniaque. Sur le principe, il n'y a guère de désaccord. On ne trouve aucun écrit médical qui ose contester la possibilité de la possession et du maléfice. Aucun écrit de juriste ou de théologien, d'autre part, ne refuse de tenir compte de la maladie naturelle et des conditions pathologiques curables par les seuls moyens naturels. La difficulté surgit lorsqu'il s'agit des critères à appliquer et de l'interprétation à donner dans les cas douteux. Faut-il voir commencer l'œuvre du diable au point où s'épuisent les ressources de la pharmacopée? Faut-il entreprendre des exorcismes dans tous les cas où le médecin perd son latin? C'est ce que pensent les théologiens. Les médecins, en revanche, déclarent que convulsions, extases, attaques de haut mal s'expliquent par les vapeurs atrabilaires montées des hypocondres, et du bas-ventre, ou par les atteintes de la substance cérébrale: ils invoquent tout à tour la puissance de l'imagination et les méfaits de la «mélancolie aduste». Mais les médecins s'accordent avec les théologiens pour déclarer que la mélancolie est le milieu propice à la pénétration de l'esprit malin; melancholia balneum diaboli; le mélancolique est une proie désignée pour le diable, et aux effets spécifiques de la dyscrasie peut s'ajouter un mal surnaturel. La grande question sera de savoir si le patient y a été voué par un charme hostile (auquel cas il convient de punir l'enchanteur maléfique), ou s'il s'y est livré de son propre gré, en cédant à son tempérament (auquel cas il en porte la faute). La victime de l'enchantement s'en tirera généralement avec des prières et des exorcismes; l'enchanteur, en revanche, est passible du feu. L'enjeu est ici d'importance. L'intervention de Johann Weyer (Wierus) (1515-1588), médecin du duc de Clèves, passe pour avoir constitué une première «révolution psychiatrique» ${ }^{18}$ : son œuvre maîtresse tendait en effet à sauver du bûcher les pauvres femmes accusées d'avoir pactisé avec le diable. A examiner le De prestigiis daemonum et incantationibus ac veneficiis libri sex (Bâle, 1563) ${ }^{19}$, l'on s'aperçoit que l'argumentation de ce médecin repose sur des considérations théologiques autant que sur des preuves médicales. Le premier livre développe une diabologie complète, et rappelle avec vigueur les thèses augustiniennes: «Le diable ne peut pas toutes choses et ne peut rien sans la permission de Dieu» (I, xxiv); il «ne saurait avoir compagnie charnelle avec une femme, ni engendrer» (ibid.); il ne peut «créer le moindre corps, ou faire quelque chose de rien», il ne peut prévoir l'avenir (I, xxv); il ne peut lire les pensées des hommes (I, xxvi). Ce diable aux pouvoirs rognés n'en 
existe pas moins; il lui arrive de se glisser dans le corps des individus. Comment agit-il? "Seules l'âme et la volonté sont corrompues par le malin esprit». Il ne confère aucun pouvoir nouveau, il ne préside pas aux métamorphoses animales des lycanthropes. Son action est purement «psychologique». C'est un illusionniste. Il impose à sa victime des prestiges insubstantiels, en l'éblouissant. C'est en rêve, dans un état d'illusion complète, que la sorcière croit assister au sabbat, s'unir charnellement au Diable, abjurer Dieu. Ces événements n'ont lieu que dans une fantaisie troublée. La théorie psychologique prend ici le relais: Weyer emprunte à Jamblique, à Ficin, à Aristote les preuves de la puissance de l'imagination. Qui ne sait que l'imagination des mélancoliques est particulièrement dépravée (livre III, vii)? Qui ne connaît la «facile croyance et fragilité du sexe féminin» (III, vi) ? Weyer allègue divers types de phénomènes hallucinatoires, sans oublier de mentionner - de source digne de foi - l'effet des toxiques: ceux qui ont absorbé du heiran luc (haschich) croient voyager dans les airs (livre III, xviii). Ainsi, là où la possession n'est pas simulée (hypothèse que Weyer envisage sérieusement), là où les aveux des sorcières ne sont pas l'effet de la torture, la sorcellerie n'est pas une action, elle est une passion. Peu importe que le diable y ait effectivement sa part: seules les actions délictueuses sont punissables. On ne saurait châtier les victimes de l'illusion. La conclusion juridique du livre de Weyer est parfaitement claire, et l'on comprend la colère de ceux qui - comme Jean Bodin - dénonçaient dans la sorcellerie une menace contre l'ordre public. (Il est difficile d'affirmer que le livre de Weyer ait eu pour effet de modérer la répression.) La thèse de Weyer est reprise en Angleterre par Reginald Scot (1599) dans The Discoverie of Witchcraft (1584). En revanche, les chimiatres disciples de Paracelse, tout en cherchant à saisir intuitivement les secrets de la nature, se montrent parfois plus crédules à l'égard de la sorcellerie, et plus enclins à donner une interprétation surnaturelle des maladies mentales ${ }^{20}$.

La pensée galénique - avec sa mise en symétrie des éléments, des qualités (chaud, sec, froid, humide), des humeurs, des tempéraments et des intempéries se propose comme un instrument explicatif cohérent, d'où découle une pratique logiquement déduite. Rien n'empêche de lier très étroitement, par des correspondances spécifiques plausibles, les diverses facultés de l'âme et les divers tempéraments. On établit de la sorte non pas une nouvelle psychologie, mais une psychologie dont la base substantielle supposée prend un aspect plus nettement déterminé. Dans l'Ejamen de los ingenios para la ciencias (Baeza, 1575), le médecin Juan Huarte de San Juan (1529-1588) fait de la sécheresse la condition du bon fonctionnement de l'entendement, affirme que la chaleur favorise l'imagination, l'humidité la mémoire : il n'échappe à l'accusation de matérialisme qu'en déclarant 
vouloir mieux définir les instruments nécessaires à la manifestation de l'âme. Ce sont toujours les mêmes facultés (entendement, imagination, mémoire), ce sont toujours les mêmes tempéraments humoraux et les mêmes qualités physiques, mais leur corrélation terme à terme est postulée de façon plus catégorique ${ }^{21}$. Affirmation toute verbale et qu'aucune véritable expérience ne soutient. Huarte croit néanmoins détenir une science assez précise pour en faire découler un programme pratique ambitieux: reconnaître les qualités dominantes d'un esprit d'après la complexion corporelle, orienter chaque individu vers le genre d'activité (d'études universitaires) qui lui convient le mieux; davantage, régler la diète et le genre de vie des parents pour qu'ils procréent des enfants remarquablement doués. Traduit dans toutes les langues européennes (Lessing en donnera une version allemande) ce livre ne doit pas son succès à l'exposé d'une psychologie différente, mais à l'affirmation d'un droit de regard de la «science», pour le bien de l'état, dans des domaines qui ne prendront que beaucoup plus tard leur dénomination moderne: psychodiagnostic, orientation professionnelle, eugénique.

Il faut aussi faire la part de l'influence néo-stoïcienne dans la psychologie de l'époque: elle est discernable dans l'attention que l'on porte à l'idéal de la constance, au rapport entre la maîtrise volontaire et la prise offerte aux forces externes (aux maladies de l'âme que sont les passions); on retrouve cette influence dans l'intérêt porté aux sympathies qui assurent l'harmonie du cosmos: l'idée d'un monde habité par une «âme» étend le domaine de la psychologie aux dimensions de l'univers. Le panthéisme s'exprime dans un panpsychisme. Rien d'étonnant si l'on prête à la matière la faculté de percevoir obscurément, ou si l'aimant passe pour manifester une sorte de volonté matérialisée, peu différente de celle qu'exercent par la vue les personnes douées du pouvoir de fascination...

A travers l'époque entière, ce qui frappe, c'est moins la révision ou le remaniement du savoir psychologique hérité des anciens, que l'extension de son champ d'application. Ce savoir ne reste pas confiné dans le milieu des doctes: il pénètre partout, il colore toutes les œuvres littéraires, il devient monnaie courante dans les manuels de conversation, il impose ses catégories (par exemple la théorie des tempéraments) à la musique, à la peinture, au ballet.

On s'attendrait à trouver des innovations chez Francis Bacon. En fait, il ne propose que des retouches et des adjonctions à la somme des certitudes relatives aux rapports de l'âme et du corps. Dans le tableau qu'il dresse des sciences manquantes (desiderata) figure une physiognomonie du corps en mouvement, une «connaissance de l'âme sensible» (c'est-à-dire du fluide corporel nécessaire à la vie animale ${ }^{22}$ ), une étude des «efforts de l'esprit [entendez: des esprits animaux] dans le mouvement volontaire», des recherches sur la lumière et sur la vision. 
Dans le domaine de l'éthique, Bacon voudrait voir se préciser les Georgica animi, science des caractères, des passions et des remèdes moraux. Loin de proposer une refonte de la psychologie traditionnelle, il l'accepte tout entière pour bâtir sur elle l'édifice des arts et des sciences. Toute l'Instauratio Magna est fondée sur la Raison, l'Imagination, la Mémoire: d'Alembert et Diderot reprendront encore, on le sait, cette partitio. Mais fonder ainsi le savoir entier sur les facultés humaines telles qu'on croit les connaître incite tôt ou tard à se demander ce que vaut une telle base. La fonction épistémologique capitale attribuée aux «facultés de l'âme» suscitera ultérieurement les critiques et les remises en question. Mais il faudra attendre le dix-neuvième siècle pour que la psychologie, cessant d'être une nomenclature hiérarchisée des facultés, se sépare (définitivement?) de la philosophie.

\section{Notes}

${ }^{1}$ Sur ces différents points de nomenclature, voir l'étude de François H. Lapointe (19).

${ }^{2}$ Voir les remarques de G. Canguilhem (6), surtout p.368-369.

${ }^{3}$ Adolphe Franck, Dictionnaire des sciences philosophiques, $3^{\mathrm{e}}$ tirage, 1885. Article Goclenius, par Emile Charles.

${ }^{4}$ Voir (5), t. II, p. 150.

${ }^{5}$ En plus du livre de Sherrington (30), on consultera aussi L. Figard, Un médecin philosophe au XVI $I^{e}$ siècle. Etude sur la psychologie de Jean Fernel. Paris, Alcan, 1903.

${ }^{6}$ Dans ses Institutiones medicae (1611), qu'on retrouve dans les Opera omnia (Venise, 1641).

7 Nous suivons l'exposé de Scipion Du Pleix.

${ }^{8}$ On trouvera un bon exposé de cette question dans le livre de Paul H.Kocher (17).

${ }^{9}$ C'est la liste proposé par Jean Fernel.

${ }^{10}$ D'où la resource d'une «thérapeutique par la parole», pratiquée par de nombreux médecins.

11 Anatomistes et physiciens s'interrogent sur les mécanismes sensoriels, et en particulier sur la vue et l'ouïe. Voir l'article très documenté de Alistair C. Crombie, "The Study of the Senses in Renaissance Science», in Actes du dixième congrès international d'histoire des sciences (Ithaca, 1962), 2 vol., Paris, 1964, t. I, p.93-117.

12 Parmi ces auteurs, citons Amatus Lusitanus (1511-?), Abraham Zacutus (1575-1642), Petrus Forestus (1522-1595).

${ }^{13}$ A ce titre, il est loué par Boerhaave, qui rééditera au $\mathrm{XVIII}^{\mathrm{e}}$ siècle le Selectiorum observationum et consiliorum de praetervisis hactenus morbis affectibusque praeter naturam, ab aqua seu serosa colluvie ortis, liber singularis (1618).

${ }^{14}$ Sur les sujets de thèses, voir 0 . Diethelm (11).

15 Nous renvoyons à l'ouvrage classique de Panofsky, Saxl et Klibansky, ainsi qu'à l'étude de D.P. Walker.

${ }^{16}$ Voir en particulier l'ouvrage de L.Babb (2). 
${ }^{17}$ Sur le rôle de la peinture de soi dans l'essor de la psychologie, voir la brève étude de Erling Eng, "The significance of the Self-Portrait and the Mirror as a Metaphor in Renaissance Developments in Psychology.» Actes du dixième congrès international d'histoire des sciences (Ithaca, 1962), 2 vol., Paris, Hermann, 1964, t. II, p. 1045-1047.

${ }^{18}$ Gregory Zilboorg a particulièrement insisté sur ce point $(38,39)$.

${ }^{19}$ La traduction française, due au poète et médecin Jacques Grévin, a été rééditée en 1885 : Histoires, disputes et discours [...], 2 vol., Paris, Delahaye et Lecrosnier.

${ }^{20}$ Voir le travail de Robert Minder (20).

${ }^{21}$ L'ouvrage d'Antonius Zara (1574-?), Anatomia ingeniorum et scientiarum (Venise, 1615) est à la fois plus largement descriptif et moins ambitieux.

${ }^{22}$ Sur ce point, Bacon se réclame ouvertement de Telesio.

${ }^{23}$ Remarquons ici que la psychologie scientifique, au sens moderne du terme, ne prendra son véritable essor qu'à partir du moment où le concept d'action et réaction (supplantant le couple antonymique action/passion) sera appliqué dans les sciences de la vie. Or, en physique, il faut d'abord avoir accepté la loi d'inertie pour pouvoir formuler le concept d'action et réaction. Ce n'est qu'au cours du XVIII ${ }^{\mathrm{e}}$ siècle que l'idée de réaction apparaîtra dans le vocabulaire des naturalistes et des "physiologistes». Cf. Jean Starobinski, «Le mot réaction: de la physique à la psychiatrie», Diogène, $\mathrm{n}^{\circ} 93,1976$, p.3-30.

\section{Bibliographie}

1. Ackerknecht, Erwin H., Kurze Geschichte der Psychiatrie. Stuttgart, F.Enke, 1957. - - A short History of Psychiatry. Transl. from the German by S. Wolff. New York/ London, Hafner, 1968.

2. Babb, Lawrence, The Elizabethan Malady. A Study of Melancholia in English Literature from 1580 to 1642. East Lansing, Michigan State University Press, 1951.

3. - - Sanity in Bedlam. A Study of Robert Burton's "Anatomy of Melancholy». East Lansing, Michigan State University Press, 1959. Bibliographie.

4. Baroja, Julio Caro, Las Brujas y su Mundo. Madrid, Revista de Occidente, 1961.

- - Les sorcières et leur monde. Traduit de l'espagnol par M.-A.Sarrailh. Paris, Gallimard, 1972.

5. Brett, George S., A History of Psychology, 3 vol. London, Allen and Unwin, 1912-1921.

- - Brett's History of Psychology, edited and abridged by R.S.Peters. London, Allen and Unwin, 1951.

6. Canguilhem, Georges, «Qu'est-ce que la psychologie», in: Etudes d'histoire et de philosophie des sciences. Paris, Vrin, 1968, p.365-381.

7. Cassirer, Ernst, Das Erkenntnisproblem, 3. Aufl. 1922/23 et 1957. Repr.: Wissenschaftliche Buchgesellschaft, Darmstadt, 1971-1973. 4 volumes.

8. Christoffel, H., «Eine systematische Psychiatrie des Barocks» [...]. Schweizer Archiv für Neurologie und Psychiatrie, Bd.77, 1956, p.15-24. Et l'on peut consulter Platter dans une traduction rècente:

Felix Platter, Observationes, I, trad. par Günther Goldschmidt, édité par Heinrich Buess, Berne et Stuttgart, Hans Huber, 1963. 
9. Diethelm, Oskar, and Hefferman, Thomas F., «Felix Platter and Psychiatry». Journal of the History of the Behavioral Sciences. Vol. I, 1965, $\mathrm{n}^{\circ}$ 1, p.10-23.

10. Diethelm, Oskar, «The medical Teaching of Demonology in the 17th and 18th Centuries». Journal of the History of the Behavioral Sciences. Vol. VI, jan. 1970, $\mathrm{n}^{\circ} 1$, p. 3-15.

11. - - Medical Dissertations of Psychiatric Interest printed before 1750. Basel, Karger, 1971.

12. Hutchings, N., "The 'Examen de Ingenios' and the Doctrine of original Genius.» Hispania, Stanford, California, XIX, 1936, p. 273-282.

12. Iriarte, Mauricio de, El Doctor Huarte de San Juan y su «Examen de Ingenios». Contribucion a la Historia de la Psicologia diferencial. Madrid, Consejo superior de Investigaciones Cientificas, 1948.

14. Karcher, Johannes, Felix Platter. Lebensbild des Basler Stadtarztes. Basel, Helbing \& Lichtenhahn, 1949.

15. Klein Robert, «L'imagination comme vêtement de l'âme chez Marsile Ficin et Giordano Bruno », in: La forme et l'intelligible. Paris, Gallimard, 1970, p.65-88.

16. Klemm, Otto, Geschichte der Psychologie. Leipzig und Berlin, Teubner, 1911.

17. Kocher, Paul H., Science and Religion in Elizabethan England. San Marino, California, The Huntington Library, 1953.

18. Laehr, Heinrich, Die Literatur der Psychiatrie, Neurologie und Psychologie von 14591799, 3 vol. Band I: Die Literatur von 1459-1699. Berlin, G. Reimer, 1900.

19. Lapointe, François H., "Who originated the Term 'Psychology'?» Journal of the History of the Behavioral Sciences. Vol. VIII, July 1972, $\mathrm{n}^{\circ} 3$, p. 328-335.

20. Minder, Robert, Der Hexenglaube bei den Iatrochemikern des 17.Jahrhunderts. Zürich, Juris, 1963. (Zürcher medizingeschichtliche Abhandlungen, Neue Reihe, $\mathrm{n}^{\mathrm{o}}$ 12.)

21. Mueller, Ferdinand-Lucien, Histoire de la Psychologie de l'antiquité à nos jours. Paris, Payot, 1960.

22. Pérouse, Gabriel-A., «Le Docteur Huarte de San Juan. Pédagogie et politique sous Philippe II ». Bibliothèque d'Humanisme et Renaissance. Genève, Droz, t. XXXII, $\mathrm{n}^{\mathrm{o}} 1,1970$, p. 81-93.

23. - - L'Examen des Esprits du Docteur Juan Huarte de San Juan. Sa diffusion et son influence en France aux XVI ${ }^{e}$ et XVII ${ }^{e}$ siècles. Paris, Les Belles Lettres, 1970. (Bibliothèque de la Faculté des Lettres de Lyon, fascicule XIX.)

24. Peset, Vicente, La psiquiatria de un medico umanista (Francisco Valles, 1524-1592). Madrid, 1961. (Paru dans Archivos de Neurobiologia, t. XXIII (1960).

25. - - «La curacion por la palabra segun Francisco Valles (1524-1592)». Cuadernos de Historia de la Medícina Española, 1964, no 3, p.3-17.

26. Rather, L. J., «Thomas Fienus (1567-1631) dialectical Investigation on Imagination as Cause and Cure of Disease». Bulletin of the History of Medicine, July-August $1967, \mathrm{n}^{\circ} 51$, p. 349-367.

27. Reimann-Hunziker, Rose, «Felix Platters Abhandlungen über die Zustände und Krankheiten des Geistes». Schweizer Archiv für Neurologie und Psychiatrie, vol.62, 1948.

28. Schüling, Hermann, Bibliographisches Handbuch zur Geschichte der Psychologie. Gießen, Universitätsbibliothek, 1964. 
29. Semelaigne, René, Les pionniers de la psychiatrie française. 2 vol., Paris, Baillière, 1932.

30. Sherrington, Charles, The endeavour of Jean Fernel. Cambridge University Press, 1946.

31. Simon, Jean-Robert, Robert Burton et l'«Anatomie de la Melancolie». Paris, Didier, 1964.

32. Spencer, Theodore, Shakespeare and the Nature of Man. Seconde éd. New York, Macmillan, 1965.

33. Temkin, Owsei, The Falling Sickness [...]. Seconde éd. Baltimore, The Johns Hopkins Press, 1971.

34. - - Galenism. Ithaca, N. Y., Cornell University Press, 1973.

35. Thorndike, Lynn, A History of Magic and Experimental Science. New York et Londres, 1923-1958, 8 vol. Vol.5-6: The sixteenth century; vol.7-8: The seventeenth century.

36. Veith, Ilza, Hysteria. The History of a Disease. Chicago, The University of Chicago Press, 1965.

37. Yates, Frances A., The Art of Memory. Londres, Routledge, 1966.

38. Zilboorg, Gregory, The Medical Man and the Witch during the Renaissance. Baltimore, Johns Hopkins Press, 1935.

39. Zilboorg, Gregory, et Henry, George W., A History of Medical Psychology. New York, Norton, 1941.

40. L'umanesimo e la "follia». Rome, Abete, 1971 (Ouvrage collectif).

41. Folie et déraison à la Renaissance. Colloque de Bruxelles, 1973. Bruxelles, Université de Bruxelles, 1976.

\section{Summary}

The author has intended to give an account of the prevalent psychological theories and of their cultural impact in Western Europe, between 1575 and 1625. The very word psychologia, which was coined during the XVIth century, has still a predominantly theological and metaphysical meaning. The medical (galenic), as well as the aristotelian traditions insist upon the reciprocal influence of body and mind. The commonly accepted doctrine is exposed after Scipion du Pleix. The somatic element taken into consideration consists in the traditional four humors and in their corruption. Although Lepois (Piso) has tried to single out the pathogenic influence of phlegm (colluvies serosa), the excess or the corruption of black bile are the most frequently alleged causes of psychic disturbances. Reciprocally, mental factors, such as passions and imagination, are equally important. The individual contributions of Johann Weyer, Felix Platter, Robert Burton, Juan Huarte de San Juan, Francis Bacon are briefly mentioned and characterized.

Prof. Dr. Jean Starobinski

12, rue de Candolle

CH-1205 Genève 